\title{
An exponential heat balance integral method
}

\author{
F. Mosally ${ }^{\text {a }}$, A.S. Wood ${ }^{\text {b,* }}$, A. Al-Fhaid ${ }^{\text {a }}$ \\ a Department of Mathematics, King Abdulaziz University, Jeddah, Saudi Arabia \\ ${ }^{\mathrm{b}}$ Department of Mechanical and Medical Engineering, University of Bradford, Bradford, \\ West Yorkshire BD7 1DP, UK
}

\begin{abstract}
Implementations of the heat balance integral method are discussed in which exponential functions are used in place of the familiar polynomial approximants. The rationale is based upon that of least-squares in that the use of 'appropriate' basis functions can enhance solution accuracy. Whilst this is true in principle it is shown that considerable skill must be exercised when deviating from polynomial approximants. The discussions are illustrated by application to a familiar single-phase Stefan problem that is typical of heat transfer problems exhibiting decay-like spatial solution profiles. (C) 2001 Elsevier Science Inc. All rights reserved.
\end{abstract}

Keywords: Heat balance integral; Stefan problem; Exponential profile

\section{Introduction}

Goodman's heat balance integral method is a semi-analytical technique for generating approximate functional solutions to, typically, transport problems that are governed by differential equations [1,2] ([3, Sections 3.5.4-3.5.6] also provides a brief discussion). The selected profile satisfies appropriate spatial boundary conditions together with an integrated form of the governing transport equation, the heat balance integral (HBI). Additional accuracy is usually obtained by spatial or temperature sub-division coupled with a lower-order piecewise approximation [4-8]. The convergence of spatial sub-division

\footnotetext{
* Corresponding author.

E-mail address: a.s.wood@bradford.ac.uk (A.S. Wood).
} 
extensions is easily observed numerically and, for simple cases, can be rigorously established $[9,10]$.

Most published papers have considered the development of polynomial profiles and in the present work the use of exponential functions is explored within the context of phase-change problems. Goodman himself [2] commented that inspecting the steady-state form of a heat transfer problem may identify an appropriate approximant for the transient phase, citing as one instance the example of a one-fluid heat exchanger (pure heat conduction) treated with an exponential profile [11]. Yang and Szewczyk [12] used a similar profile to treat pure heat conduction with variable material properties.

Many 'source term' problems in heat conduction, including phase-change problems, admit 'decaying' exponential-type solution profiles and here we investigate the use of several polynomial and exponential HBI implementations, both for whole-domain solutions and for piecewise solutions using spatial subdivision. It will be seen that whilst the application of piecewise linear forms essentially requires a mechanistic approach, the use of piecewise exponential approximants is somewhat more of an 'art'. The reward, in terms of accuracy, for an appropriate exponential selection is considerable, although it appears that little can compare with the ease and general applicability of polynomial approximation.

\section{A model problem}

To avoid unnecessary algebraic complications in the discussions we consider the following one-phase problem that describes the (dimensionless) melting of a solid semi-infinite material initially at its melt temperature:

$$
\begin{aligned}
& \frac{\partial u}{\partial t}=\frac{\partial^{2} u}{\partial x^{2}}, \quad 0<x<s, \quad t>0 \\
& \frac{\partial u}{\partial x}=-\beta \frac{\mathrm{d} s}{\mathrm{~d} t}, \quad x=s(t), \quad t>0 \\
& u=0, \quad x>0, \quad t=0 \\
& u=1, \quad x=0, \quad t \geqslant 0 \\
& u=0, \quad x=s(t), \quad t>0
\end{aligned}
$$

The well-known analytical solution to this problem is [13, Section 11.2] 


$$
\begin{aligned}
& u(x, t)=1-\frac{\operatorname{erf}(x / 2 \sqrt{t})}{\operatorname{erf}(\alpha)}, \quad 0 \leqslant x \leqslant s(t), \quad t \geqslant 0, \\
& s(t)=2 \alpha \sqrt{t}, \quad t \geqslant 0,
\end{aligned}
$$

where $u$ denotes temperature, $s$ denotes the location of the melt front and $\alpha$ is the solution of the transcendental equation $\sqrt{\pi} \beta \alpha \operatorname{erf}(\alpha) \mathrm{e}^{\alpha^{2}}=1 . x$ and $t$ denote the usual independent space and time variables.

\section{Whole-domain HBI solutions}

The basic implementation of the HBI method generates a single functional approximation that is defined for the entire spatial solution domain - a wholedomain solution. Here the classical quadratic polynomial solution is outlined followed by two exponential solutions.

\subsection{Classical polynomial HBI approximation}

The quadratic approximant used by Goodman [2, p. 73] took the form $v(x, t)=b(x-s)+c(x-s)^{2}$ where the melt front $s$ and the parameters $b$ and $c$ (functions of time) are obtained by enforcing the spatial conditions (2) and (4) together with a heat balance integral that is obtained by integrating Eq. (1) over the interval $0 \leqslant x \leqslant s$ and replacing $u$ by $v$. Condition (5) is already satisfied. Goodman [2] used the alternative form for Eq. (2)

$$
\left(\frac{\partial u}{\partial x}\right)^{2}=\beta \frac{\partial^{2} u}{\partial x^{2}}, \quad x=s(t), t>0,
$$

(which shows the non-linear nature of the melting problem) and suggested that its use avoids the introduction of a second-order differential equation for $s$. In fact appropriate use of Eq. (2) does not introduce higher-order derivatives and also produces a more accurate solution [14].

The quadratic form

$$
v(x, t)=b\left(1-\frac{x}{s}\right)+c\left(1-\frac{x}{s}\right)^{2},
$$

admits parameters $b$ and $c$ that are constants. Conditions (2) and (4) combine with the heat balance integral

$$
\int_{0}^{s} \frac{\partial v}{\partial t} \mathrm{~d} x=-\beta \frac{\mathrm{d} s}{\mathrm{~d} t}-\left.\frac{\partial v}{\partial x}\right|_{x=0}
$$

to produce the equations 
Table 1

Analytic, quadratic HBI and exponential HBI solutions

\begin{tabular}{lllll}
\hline$x / s$ & Analytic & $\begin{array}{l}\text { Quadratic } \\
\text { Eq. (9) }\end{array}$ & Exponential & \\
\cline { 4 - 5 } & & 1 & 1 & Eq. (11) \\
\hline 0.0 & 1 & 0.7754 & 0.7753 & 1 \\
0.2 & 0.7753 & 0.5652 & 0.5666 & 0.7756 \\
0.4 & 0.5573 & 0.3694 & 0.3730 & 0.5575 \\
0.6 & 0.3523 & 0.1880 & 0.1932 & 0.3518 \\
0.8 & 0.1654 & 0.0210 & 0.0262 & 0.1638 \\
1.0 & 0 & 1.2730 & 1.2808 & -0.0018 \\
$s$ & 1.2402 & & & 1.2371 \\
\hline
\end{tabular}

$$
s \frac{\mathrm{d} s}{\mathrm{~d} t}=\frac{b}{\beta}, \quad b+c=1, \quad \text { and } \quad s \frac{\mathrm{d} s}{\mathrm{~d} t}=\frac{6(b+2 c)}{3 b+2 c+6 \beta} .
$$

Eliminating $c$ and $s(\mathrm{~d} s / \mathrm{d} t)$ gives an equation for $b, b^{2}+(2+12 \beta) b-12 \beta=0$, with solution $b=-1-6 \beta+\sqrt{1+24 \beta+36 \beta^{2}}$. Thus

$$
s=2 \sqrt{\frac{b t}{2 \beta}}=2 \alpha^{*} \sqrt{t}, \quad \alpha^{*}=\sqrt{\frac{-1-6 \beta+\sqrt{1+24 \beta+36 \beta^{2}}}{2 \beta}} .
$$

For $\beta=1$ the parameter values are $b=0.8102, c=0.1898$ and $\alpha^{*}=0.6365$. The solution is shown in Table 1 for $t=1$.

\subsection{Exponential HBI approximations}

The test problem (1)-(5) does not have a finite-domain steady-state solution. The characteristic process of heat transfer (Fourier's law) suggests that a suitable transient approximant might be exponential,

$$
v(x, t)=a+b \mathrm{e}^{c x / s},
$$

where $a, b$, and $c$ are constants to be determined. Conditions (2), (4) and (5) combine with the heat balance integral (10) to give

$$
\begin{aligned}
& s \frac{\mathrm{d} s}{\mathrm{~d} t}=-\frac{b c \mathrm{e}^{c}}{\beta}, \quad a+b=1, \quad a+b \mathrm{e}^{c}=0, \\
& s \frac{\mathrm{d} s}{\mathrm{~d} t}=\frac{b c^{2}}{b\left(c \mathrm{e}^{c}-\mathrm{e}^{c}+1\right)-\beta c} .
\end{aligned}
$$

Eliminating $a, b$ and $s(\mathrm{~d} s / \mathrm{d} t)$ yields $[(1+\beta) c-1] \mathrm{e}^{2 c}-(2 \beta c-1) \mathrm{e}^{c}+\beta c=0$ that may be solved for $c$. $a, b$ and $s=2 \alpha^{*} \sqrt{t}$ are found from Eq. (12), where 


$$
\alpha^{*}=\sqrt{-\frac{b c \mathrm{e}^{c}}{2 \beta}}=\sqrt{\frac{c \mathrm{e}^{c}}{2\left(\mathrm{e}^{c}-1\right) \beta}} .
$$

For $\beta=1, c=-0.3840, b=3.1364, a=-2.1364$ and $\alpha^{*}=0.6404$. The solution is listed in column 4 of Table 1 for $t=1$. A cursory inspection of the tabulated values appears to contradict the original suggestion that an exponential profile might provide more accuracy than a quadratic profile. The values simply confirm the acknowledged sensitivity of the basic method to the form of the approximant. To emphasise this consider the three-parameter exponential approximant

$$
v(x, t)=a+\frac{b x}{s} \mathrm{e}^{c x^{2} / s^{2}} .
$$

Conditions (2), (4) and (5) together with HBI (10) generate

$$
\begin{aligned}
& s \frac{\mathrm{d} s}{\mathrm{~d} t}=-\frac{b \mathrm{e}^{c}(1+2 c)}{\beta}, \quad a=1, \quad a+b \mathrm{e}^{c}=0, \\
& s \frac{\mathrm{d} s}{\mathrm{~d} t}=-\frac{2 b c}{b\left(\mathrm{e}^{c}-1-2 c \mathrm{e}^{c}\right)+2 c \beta} .
\end{aligned}
$$

The equation $(1+2 c)\left[2 c \mathrm{e}^{c}(1+\beta)-\mathrm{e}^{c}+1\right]=2 c \beta$ is obtained by eliminating $a$, $b$ and $s(\mathrm{~d} s / \mathrm{d} t)$. For $\beta=1, c=-0.1174, b=-1.1245$ and $\alpha^{*}=0.6186$. Solution (15) is tabulated in column 5 of Table 1 and shows a remarkable improvement in accuracy. $x \mathrm{e}^{-x^{2}}$ describes the first term in the power series of erf $x$ and the ensuing results serve to confirm the earlier suggestion that 'considerable skill must be exercised' when selecting non-polynomial approximants, a choice that will be problem dependant. Fig. 1 reinforces these sentiments with respect to the melt front, and Fig. 2 shows the spatial distribution of the absolute error (in magnitude).

\section{Two-parameter piecewise HBI solutions}

Higher accuracy is achieved by the well-established route of domain decomposition [4]. Here the use of piecewise exponential forms is compared with the piecewise linear form.

The domain $[0, s]$ is decomposed into $n$ equal sub-intervals of length $s / n$ and a two-parameter profile is developed for each sub-interval. With the melt front, $s, 2 n+1$ unknowns are introduced. On each sub-interval an integral form of Eq. (1) is satisfied by the selected function together with continuity at the subinterval junctions. 


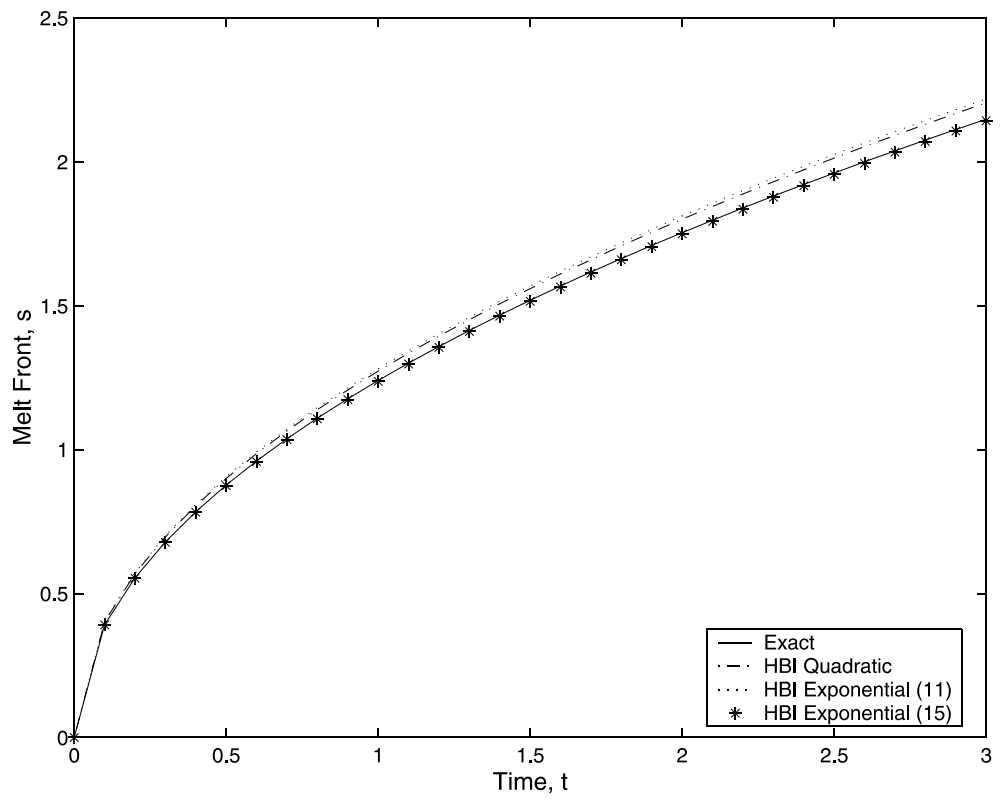

Fig. 1. Predicted melt front history.

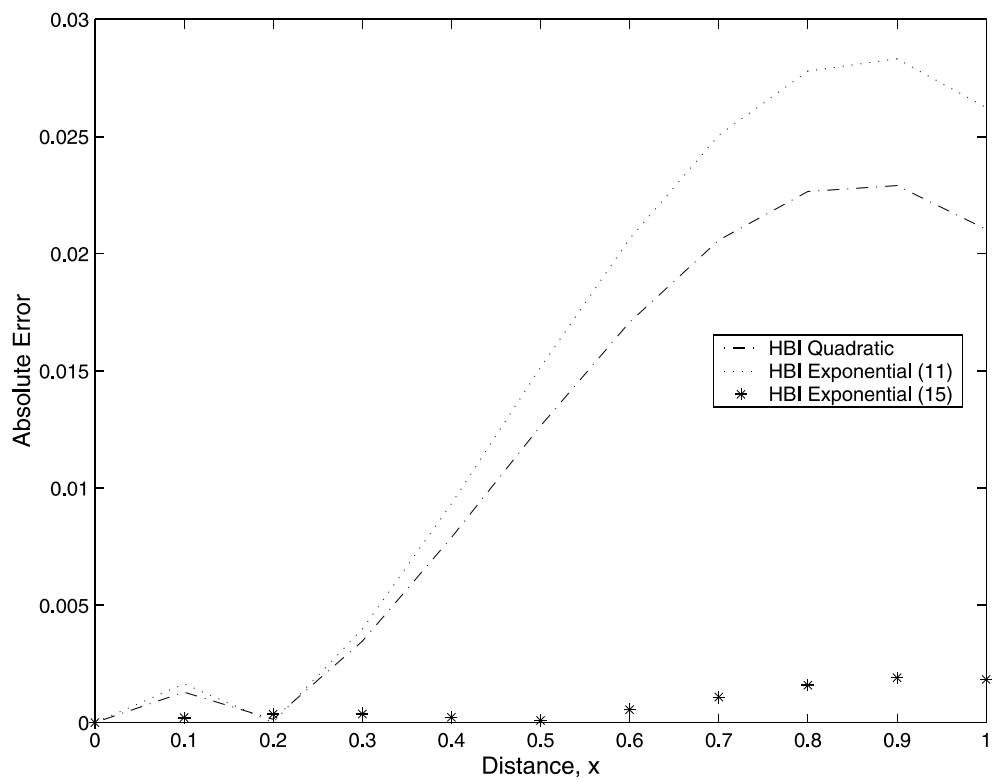

Fig. 2. Absolute error in the temperature profile predictions at $t=1$. 


\subsection{Piecewise linear approximation [10]}

Let $v_{i} \simeq u\left(x_{i}, t\right), v_{0}=1$ and $v_{n}=0$, where $x_{i}=i s / n$, and define $v=a_{i}+b_{i} x$ for $x_{i} \leqslant x \leqslant x_{i+1}$. In finite-element parlance $a_{i}$ and $b_{i}$ are generalised coordinates of which there are $2 n$. To reduce the number of unknowns to $n-1$ we may recast the elemental approximants in terms of the nodal variables $v_{0}, \ldots, v_{n}$,

$$
v=v_{i}+\left(x-x_{i}\right)\left(v_{i+1}-v_{i}\right) \frac{n}{s}, \quad x_{i} \leqslant x \leqslant x_{i+1}, \quad i=0, \ldots, n-1 .
$$

The nodal temperature gradients are taken to be the piecewise constants

$$
\frac{\partial v}{\partial x}=\frac{n}{s}\left(v_{i+1}-v_{i}\right), \quad x=x_{i}, \quad i=0, \ldots, n-1,
$$

with

$$
\frac{\partial v}{\partial x}=-\beta \frac{\mathrm{d} s}{\mathrm{~d} t} \text { at } x=x(t) .
$$

A heat balance integral is generated on each sub-interval from Eq. (1)

$$
\int_{x_{i}}^{x_{i+1}} \frac{\partial v}{\partial t} \mathrm{~d} x=\left[\frac{\partial v}{\partial x}\right]_{x=x_{i}}^{x=x_{i+1}} .
$$

Substituting Eqs. (16) and (17) into (18) yields $n$ ODEs

$$
\begin{aligned}
& s \frac{\mathrm{d} s}{\mathrm{~d} t}=\frac{2 n^{2}}{2 i+1} \frac{v_{i+2}-2 v_{i+1}+v_{i}}{v_{i}-v_{i+1}}, \quad i=0, \ldots, n-2, \\
& s \frac{\mathrm{d} s}{\mathrm{~d} t}=\frac{2 n^{2} v_{n-1}}{(2 n-1) v_{n-1}+2 n \beta}, \quad i=n-1 .
\end{aligned}
$$

Equating the right-hand sides of (19) and (20), $v_{i}$ can be expressed as

$$
v_{i}=v_{i+1}-f_{i}\left(v_{i+2}-v_{i+1}\right), \quad i=0, \ldots, n-2,
$$

where $f_{i}=(n+\eta-1 / 2) /(n+\eta-i-1)$ and $\eta=n \beta / v_{n-1}$. Repeated application of Eq. (21), with $v_{0}=1$, produces a single equation in $\eta$,

$$
1-\frac{\eta}{n}+\sum_{i=1}^{n-1} \frac{(n+\eta-1 / 2)^{i}}{(\eta+i)(\eta+i-1) \ldots(\eta+1)}=0 .
$$

On solving Eq. (22) for $\eta$, the approximate temperature is found from $v_{n-1}=$ $n \beta / \eta$ and Eq. (21), and the melt front is obtained from Eq. (20),

$$
s=2 \alpha^{*} \sqrt{t}, \quad \alpha^{*}=\frac{n}{\sqrt{2 n+2 \eta-1}} .
$$

Convergence to the analytical solution is observed with increasing $n$ (see Table 2) and may be confirmed rigorously [10]. The value $v_{i}$ is interpreted as an estimate to $u\left(x_{i}, t\right)$. For $\beta=1$ and $n=2$, Eq. (22) becomes $\eta^{2}-3 \eta-5=0$ with solution $\eta=4$.1926. The remaining solution parameters are $v_{1}=0.4770$ and 
Table 2

Convergent piecewise linear HBI solution

\begin{tabular}{lllll}
\hline$x / s$ & \multicolumn{2}{l}{ Piecewise linear } & & Analytic \\
\cline { 2 - 4 } & $n=10$ & $n=20$ & $n=40$ & \\
\hline 0.0 & 1 & 1 & 1 & 1 \\
0.2 & 0.7785 & 0.7769 & 0.7761 & 0.7753 \\
0.4 & 0.5619 & 0.5596 & 0.5585 & 0.5573 \\
0.6 & 0.3566 & 0.3545 & 0.3534 & 0.3523 \\
0.8 & 0.1680 & 0.1666 & 0.1660 & 0.1654 \\
1.0 & 0 & 0 & 0 & 0 \\
$s$ & 1.2279 & 1.2339 & 1.2370 & 1.2402 \\
\hline
\end{tabular}

$\alpha^{*}=0.5927(s \approx 1.1855)$, and the resulting temperature profile at $t=1$, shown in Fig. 3, is remarkably good.

\subsection{Piecewise exponential approximations}

A natural choice of exponential approximation to $u$ is

$$
v(x, t)=a_{i}+b_{i} \mathrm{e}^{-x / s}, \quad x_{i-1} \leqslant x \leqslant x_{i}, \quad i=1, \ldots, n,
$$

where $a_{i}$ and $b_{i}$ are constants. Using the boundary conditions (4) and (5), and enforcing continuity at $x_{i}=i s / n$,

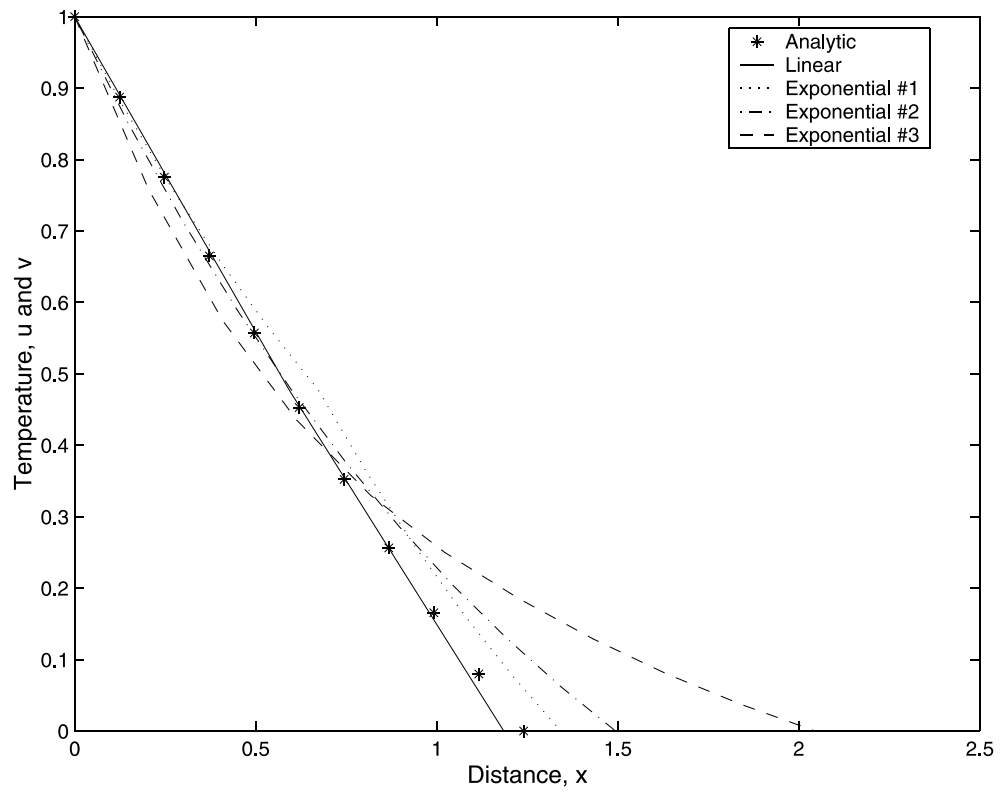

Fig. 3. Two-element piecewise temperature profiles at $t=1$. 


$$
\begin{aligned}
& a_{1}=1-b_{1}, \quad a_{n}=-b_{n} \mathrm{e}^{-1}, \\
& a_{i+1}=a_{i}+\mathrm{e}^{-\mathrm{i} / n}\left(b_{i}-b_{i+1}\right), \quad i=1, \ldots, n-1 .
\end{aligned}
$$

Eqs. (25) and (26) can be combined to give

$$
1+\sum_{i=1}^{n} b_{i}\left(\mathrm{e}^{-\mathrm{i} / n}-\mathrm{e}^{-(\mathrm{i}-1) / n}\right)=0 .
$$

Replacing $u$ by $v$, Eq. (24), in Eq. (1) and integrating over each sub-interval $\left[x_{i-1}, x_{i}\right]$ with respect to $x$, taking the temperature gradients as

$$
\frac{\partial v}{\partial x}=-\frac{b_{i+1}}{s} \mathrm{e}^{-\mathrm{i} / n}, \quad x=x_{i}, \quad i=0, \ldots, n-1
$$

with

$$
\frac{\partial v}{\partial x}=-\beta \frac{\mathrm{d} s}{\mathrm{~d} t} \text { at } x=s(t)
$$

give

$$
\begin{array}{ll}
s \frac{\mathrm{d} s}{\mathrm{~d} t}=\frac{n\left(\mathrm{e}^{1 / n} b_{i}-b_{i+1}\right)}{b_{i}\left[\mathrm{e}^{1 / n}(n+i-1)-(n+i)\right]}, & i=1, \ldots, n-1, \\
s \frac{\mathrm{d} s}{\mathrm{~d} t}=\frac{n b_{n} \mathrm{e}^{1 / n}}{b_{n}\left[(2 n-1) \mathrm{e}^{1 / n}-2 n\right]+\beta e n}, & i=n .
\end{array}
$$

Equating the right-hand sides of Eqs. (29) and (30) produces

$$
\begin{aligned}
& b_{n} \mathrm{e}^{1 / n} b_{i}\left[(i+n-1) \mathrm{e}^{1 / n}-(i+n)\right]-\left(\mathrm{e}^{1 / n} b_{i}-b_{i+1}\right) \\
& \quad\left[\beta e n+b_{n}\left[(2 n-1) \mathrm{e}^{1 / n}-2 n\right]\right]=0, \quad i=1, \ldots, n-1 .
\end{aligned}
$$

Eqs. (27) and (31) form a system of $n$ non-linear equations with $n$ unknowns $b_{1}, \ldots, b_{n}$ (readily solved using Newton's method). The $a_{i}$ are found from Eq. (26).

A predictable drawback of form (24) is that the decay term $\mathrm{e}^{-x / s}$ is the same for each sub-interval (unlikely in practice). For $\beta=1$ and $n=2$ we obtain $b_{2}=2.0063, b_{1}=1.3246, a_{2}=-0.7381, a_{1}=-0.3246$ and $\alpha^{*}=0.6715(s \approx$ 1.3431). Fig. 3 (curve 'Exponential \#1') confirms the poor quality of the approximation - a 'kink' (first-derivative discontinuity) may be observed at approximately $x=0.65$ together with an inaccurate estimate of the melt front. The situation is improved by increasing $n$ but the piecewise exponential form never recovers its poor performance as compared to the piecewise linear form.

To ensure first-derivative continuity at the nodes $x_{i}$ requires, from elementary calculus, that $b_{1}=\cdots=b_{i}=\cdots=b_{n}$. For the case $n=2, b_{1}=b_{2}=b$ and continuity at $x=s / 2$ gives $b=e /(e-1) \approx 1.5820$. This leaves just one 
unknown, $s$, and so we cannot expect to satisfy two elemental HBIs. Consequently a whole-domain HBI is developed to give

$$
s \frac{\mathrm{d} s}{\mathrm{~d} t}=\frac{b}{b\left(1-2 \mathrm{e}^{-1}\right)+\beta}
$$

with solution $s=2 \alpha^{*} \sqrt{t}$, where

$$
\alpha^{*}=\sqrt{\frac{b}{2\left[b\left(1-2 \mathrm{e}^{-1}\right)+\beta\right]}} .
$$

With $\beta=1, \alpha^{*}=0.7469(s \approx 1.4937)$ and the solution profile is shown in Fig. 3 (curve 'Exponential \#2'). There is some improvement over curve \#1 for the first half of the domain, but the imposition of first-derivative continuity has swung the melt front away from the analytical value.

An alternative two-parameter exponential approximant that incorporates varying decay rates takes the form

$$
v(x, t)=a_{i}+\mathrm{e}^{c_{i} x / s}, \quad x_{i-1} \leqslant x \leqslant x_{i}, \quad i=1, \ldots, n .
$$

Following the process described for the form (24) results in $n$ non-linear equations for $c_{1}, \ldots, c_{n}$. This form fairs no better - for $\beta=1$ and $n=2$, curve \#3 in Fig. 3 shows the worsening approximation.

\section{Three-parameter piecewise HBI solutions}

The attraction of two-parameter approximations is that each additional subinterval introduces two further unknowns that can be determined by simply enforcing continuity at the sub-interval extrema and satisfying an additional elemental HBI. Given the exceptional results obtained with function (15) we conclude with a brief investigation of some three-parameter piecewise HBI implementations.

In general terms $n$ sub-intervals introduce $3 n+1$ unknowns ( $3 n$ profile parameters plus the interface location $s$ ). The following set of conditions is used to generate the necessary equations:

\begin{tabular}{ll}
\hline Conditions & No. equations \\
\hline Boundary conditions (4) and (5) & 2 \\
Continuity at $x_{i}$ & $n-1$ \\
Slope continuity at $x_{i}$ & $n-1$ \\
HBI equations & $n$ \\
Stefan condition (2) & 1 \\
Total & $3 n+1$ \\
\hline
\end{tabular}


Three approximation forms are considered,

$$
v(x, t)=\left\{\begin{array}{cl}
a_{i}+b_{i} \frac{x}{s}+c_{i} \frac{x^{2}}{s^{2}}, & \text { quadratic, } \\
a_{i}+b_{i} \mathrm{e}^{c_{i} x / s}, & \text { exponential, } \\
a_{i}+\frac{b_{i} x}{s} \mathrm{e}^{c_{i} x^{2} / s^{2}}, & \text { Gaussian }
\end{array}\right.
$$

and two-element solutions are developed for each form to illustrate their relative merits. In this case 7 equations are required to evaluate the 7 unknowns $a_{1}, b_{1}, c_{1}, a_{2}, b_{2}, c_{2}$ and $\alpha$ (i.e., s). Conditions (4) and (5) serve to determine $a_{1}$ and $a_{2}$. The values are $a_{1}=1$ and $a_{2}=-b_{2}-c_{2}$ (quadratic), $a_{1}=1-b_{1}$ and $a_{2}=-b_{2} \mathrm{e}^{c_{2}}$ (exponential), and $a_{1}=1$ and $a_{2}=-b_{2} \mathrm{e}^{c_{2}}$ (Gaussian). Implementing the remaining five conditions generates equations for the outstanding parameters:

- Quadratic:

$$
\begin{aligned}
& 4+2 b_{1}+c_{1}=-2 b_{2}-3 c_{2}, \\
& b_{1}+c_{1}=b_{2}+c_{2}, \\
& 24\left(b_{2}+c_{2}-b_{1}\right) \beta=\left(b_{2}+2 c_{2}\right)\left(3 b_{1}+2 c_{1}\right), \\
& 24\left(b_{2}+c_{2}\right) \beta=\left(b_{2}+2 c_{2}\right)\left(-9 b_{2}-14 c_{2}+24 \beta\right), \\
& s \frac{\mathrm{d} s}{\mathrm{~d} t}=-\frac{b_{2}+2 c_{2}}{\beta} .
\end{aligned}
$$

- Exponential:

$$
\begin{aligned}
& 1+b_{1}\left(\mathrm{e}^{c_{1} / 2}-1\right)=b_{2}\left(\mathrm{e}^{c_{2} / 2}-\mathrm{e}^{c_{2}}\right), \\
& b_{1} c_{1} \mathrm{e}^{c_{1} / 2}=b_{2} c_{2} \mathrm{e}^{c_{2} / 2}, \\
& \left(b_{1} c_{1}-b_{2} c_{2} \mathrm{e}^{c_{2} / 2}\right) \beta=-b_{1} b_{2} c_{2} \mathrm{e}^{c_{2}}\left[\frac{\mathrm{e}^{c_{1} / 2}}{2}-\frac{\mathrm{e}^{c_{1} / 2}-1}{c_{1}}\right], \\
& \mathrm{e}^{c_{2} / 2} \beta=-\mathrm{e}^{c_{2}}\left\{b_{2}\left[\mathrm{e}^{c_{2}}-\frac{\mathrm{e}^{c_{2} / 2}}{2}-\frac{\mathrm{e}^{c_{2}}-\mathrm{e}^{c_{2} / 2}}{c_{2}}\right]-\beta\right\}, \\
& s \frac{\mathrm{d} s}{\mathrm{~d} t}=-\frac{b_{2} c_{2} \mathrm{e}^{c_{2}}}{\beta} .
\end{aligned}
$$


- Gaussian:

$$
\begin{aligned}
& 2+b_{1} \mathrm{e}^{c_{1} / 4}=b_{2}\left(\mathrm{e}^{c_{2} / 4}-2 \mathrm{e}^{c_{2}}\right), \\
& b_{1} \mathrm{e}^{c_{1} / 4}\left(2+c_{1}\right)=b_{2} \mathrm{e}^{c_{2} / 4}\left(2+c_{2}\right), \\
& \left(2 b_{1}-b_{2} \mathrm{e}^{c_{2} / 4}\left(2+c_{2}\right)\right) \beta=-b_{1} b_{2} \mathrm{e}^{c_{2}}\left(1+2 c_{2}\right)\left[\frac{\mathrm{e}^{c_{1} / 4}}{2}-\frac{\mathrm{e}^{c_{1} / 4}-1}{c_{1}}\right], \\
& \mathrm{e}^{c_{2} / 4}\left(2+c_{2}\right) \beta=-\mathrm{e}^{c_{2}}\left(1+2 c_{2}\right)\left\{b_{2}\left[2 \mathrm{e}^{c_{2}}-\frac{\mathrm{e}^{c_{2} / 4}}{2}-\frac{\mathrm{e}^{c_{2}}-\mathrm{e}^{c_{2} / 4}}{c_{2}}\right]-2 \beta\right\}, \\
& s \frac{\mathrm{d} s}{\mathrm{~d} t}=-\frac{b_{2} \mathrm{e}^{c_{2}}\left(1+2 c_{2}\right)}{\beta} .
\end{aligned}
$$

The various systems of equations are numerically tractable (e.g., Newton's method) and once the coefficients $b_{i}$ and $c_{i}$ have been determined the estimates $\alpha^{*}$ may be computed. For $\beta=1$ the three approximations give the values 0.6238 (error 0.0037), 0.6251 (error 0.0050) and 0.6195 (error 0.0006). All estimates are very accurate. The quadratic (polynomial) again outperforms the exponential form and the high accuracy obtained from the Gaussian form extends to the piecewise implementation. Fig. 4 shows the spatial error dis-

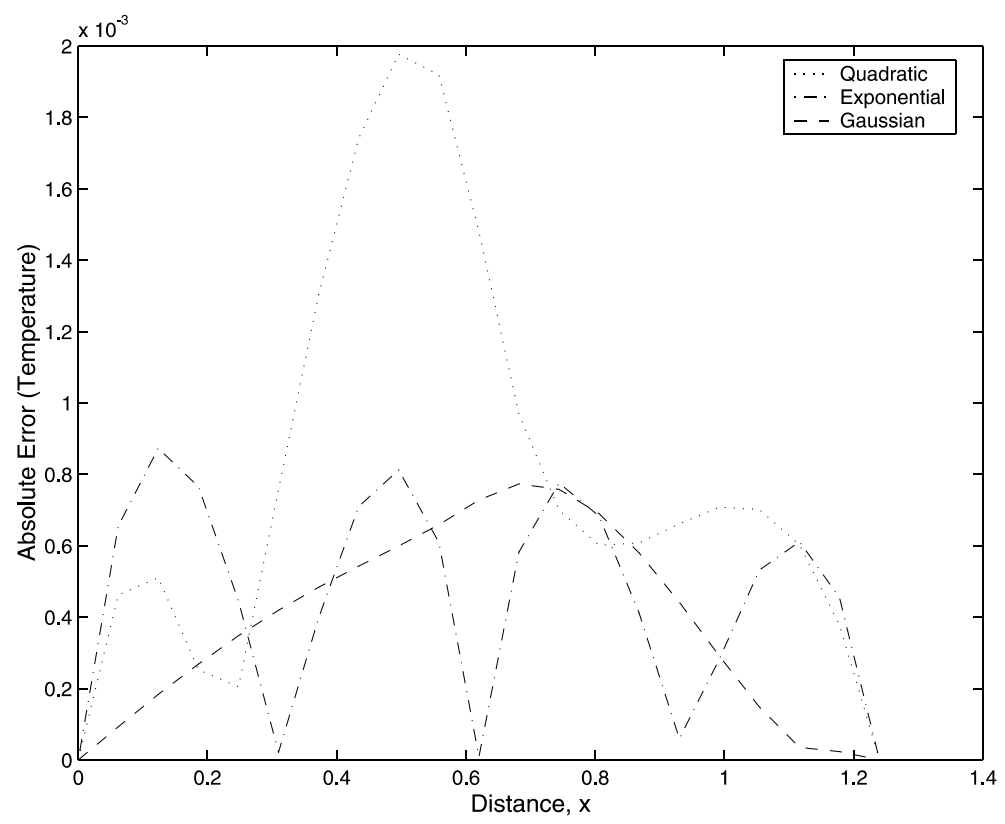

Fig. 4. Two-element three-parameter temperature error profiles at $t=1$. 
tribution for the temperature estimates given by the three approximations (the temperature curves are virtually coincident). The exponential error curve exhibits an undesirable oscillatory behaviour indicating that the solution curve criss-crosses the analytical solution.

\section{Conclusions}

The policy of incorporating 'user knowledge' to implement HBI solutions other than polynomial is not straightforward. A very sound understanding is required of the underlying functions governing the (temperature) profiles for any particular problem - it was evidently not sufficient to assume that the solution of the model problem (1)-(5) 'behaves like exponential decay'. Nonetheless, identifying realistic functions, e.g., $x \mathrm{e}^{-x^{2}}$ pays immense dividends in terms of solution accuracy.

Evidently there is little to rival the piecewise linear approximations in terms of low computational effort (a single non-linear equation) and general applicability, supporting observations of previous authors [9]. However, for highly accurate solutions the basic appropriate approximant (in this case $a+b \mathrm{e}^{c x^{2} / s^{2}}$ ) can be extended to a piecewise form. Modern software readily facilitates the solution of the resulting non-linear systems of algebraic equations.

\section{References}

[1] T.R. Goodman, The heat-balance integral and its application to problems involving a change of phase, Trans. ASME J. Heat Transfer 80 (1958) 335-342.

[2] T.R. Goodman, Application of integral methods in transient non-linear heat transfer, in: T.F. Irvine, J.P. Hartnett (Eds.), Advances in Heat Transfer, vol. 1, Academic Press, New York, 1964, pp. 51-122.

[3] J. Crank, Free and Moving Boundary Problems, Clarendon Press, Oxford, 1984.

[4] B. Noble, Heat balance methods in melting problems, in: J.R. Ockendon, W.R. Hodgkins (Eds.), Moving Boundary Problems in Heat Flow and Diffusion, Clarendon Press, Oxford, 1974, pp. 208-209.

[5] G.E. Bell, A refinement of the heat balance integral method applied to a melting problem, Int. J. Heat Mass Transfer 21 (1978) 1357-1362.

[6] G.E. Bell, Solidification of a liquid about a cylindrical pipe, Int. J. Heat Mass Transfer 22 (1979) 1681-1686.

[7] G.E. Bell, The accurate solution of one-dimensional melting problems by the heat balance integral method, in: R.W. Lewis, K. Morgan, (Eds.), Numerical Methods in Thermal Problems, Pineridge Press, Swansea, 1979, pp. 196-203.

[8] G.E. Bell, The prediction of frost penetration, Int. J. Numer. Anal. Meth. Geomech. 6 (1982) 287-290.

[9] G.E. Bell, S.K. Abbas, Convergence properties of the heat balance integral method, Numer. Heat Transfer 8 (1985) 373-382.

[10] F. Mosally, A.S. Wood, A. Al-Fhaid, On the convergence of the heat balance integral method applied to a phase-change problem, Free and Moving Boundaries, submitted. 
[11] W.C. Reynolds, T.A. Dolton, Use of integral methods in transient heat transfer analysis, ASME Paper No. 58-A-248, 1958.

[12] K.T. Yang, A. Szewczyk, An approximate treatment of unsteady heat conduction in semiinfinite solids with variable thermal properties, Trans. ASME J. Heat Transfer 81 (1959) 251252.

[13] H.S. Carslaw, J.C. Jaeger, Conduction of Heat in Solids, second ed., Clarendon Press, Oxford, 1959.

[14] A.S. Wood, A new look at the heat balance integral method, Appl. Math. Modelling 25 (2001) 815-824. 